\title{
Should rare immunologic, neurologic, and other adverse events be indications to withhold vaccination?
}

\author{
Donald E. Greydanus ${ }^{1}$, Andrey Leonov ${ }^{1,2}$, Ahmed Elisa ${ }^{1}$, Roua Azmeh ${ }^{1}$ \\ ${ }^{1}$ Department of Pediatric \& Adolescent Medicine, Western Michigan University Homer Stryker M.D. School of Medicine, Kalamazoo, MI, USA; \\ ${ }^{2}$ DuPage Medical Group, Plainfield, IL, USA \\ Contributions: (I) Conception and design: All authors; (II) Administrative support: None; (III) Provision of study materials or patients: None; (IV) \\ Collection and assembly of data: None; (V) Data analysis and interpretation: None; (VI) Manuscript writing: All authors; (VII) Final approval of \\ manuscript: All authors. \\ Correspondence to: Roua Azmeh. Department of Pediatric \& Adolescent Medicine, Western Michigan University Homer Stryker M.D. School of \\ Medicine, 1000 Oakland Drive, Kalamazoo, MI 49008, USA. Email: roua.azmeh@med.wmich.edu.
}

\begin{abstract}
Millions of illnesses, hospitalizations, and deaths are prevented by vaccination worldwide. This discussion examines vaccine safety concerns from the past several decades of immunization research. Both immunologic and non-immunologic side effects are reviewed, and clarification is provided regarding some highly-publicized myths regarding vaccine safety.
\end{abstract}

Keywords: Adverse effects; fear; immunization; vaccines

Submitted May 16, 2019. Accepted for publication Jun 10, 2019.

doi: $10.21037 /$ tp.2019.06.01

View this article at: http://dx.doi.org/10.21037/tp.2019.06.01

\section{Introduction}

Research shows that millions of illnesses, hospitalizations, and deaths have been prevented by widespread application of vaccine science (1). Despite this success in vaccineprevented morbidity and mortality, concerns about the effectiveness and safety of immunizations have continued to echo since the dawn of vaccine development $(2,3)$. Within the last few years, vaccine hesitancy has risen to such a level as to be identified by the World Health Organization as one of the top 10 threats to global health in 2019 (4). With vaccine hesitancy comes declining vaccination rates, which threatens the herd immunity that normally prevents extremely communicable diseases from easily spreading to cause outbreaks. Diseases such as measles which were once eliminated in many parts of the world have caused several outbreaks in the US and other countries across the globe, frequently amongst pockets of unvaccinated people who may live or work together and share religious or cultural values $(5,6)$. In the midst of publicized myths about vaccineinduced disease, and despite their overall favorable safety profile, there are rare but potentially severe adverse effects linked with vaccines that healthcare workers should be aware of as they advise their patients. This article discusses aspects of vaccine safety through the lens of the past several decades of immunization research.

\section{Immunologic adverse events}

\section{Hypersensitivity}

Immunologic reactions, including anaphylaxis, can be triggered by multiple vaccine components (7). Immunemediated reactions to vaccines can be immediate (i.e., within minutes of antigen exposure) or delayed (i.e., hours to days post-exposure). Immediate-type reactions are typically Gell and Coombs Type 1 IgE-mediated allergic reactions, whereas delayed-type are usually not IgE-mediated [i.e., Type 3 small immune complex (IgG)-mediated reactions associated with complement activation such as Arthus reactions or serum sickness, or Type 4 T-cell-mediated allergic reactions with multiple presentations ranging from systemic contact dermatitis to Stevens-Johnson syndrome/ toxic epidermal necrolysis] (8). Diphtheria toxoid-tetanus 
toxoid-acellular pertussis ( $\mathrm{DTaP})$ is well known to be associated with large local limb swelling after injections, especially after a patient has received several doses of the recommended series. This has been postulated to be an example of Arthus reaction, but evidence is conflicting and there may be multiple factors responsible $(9,10)$.

Potential allergenic agents in vaccines that can induce $\mathrm{IgE}$-mediated reactions include culture-related contaminants such as ovalbumin (in vaccines cultured in hen's egg), yeast proteins (in vaccines cultured in Saccharomyces cerevisiae), or antibiotics (mainly neomycin but also polymyxin $\mathrm{B}$, streptomycin, gentamicin, amphotericin $\mathrm{B}$, and/or chlortetracycline). Of note, hen's egg as a culture medium was previously thought to cause ovalbumin contamination of influenza vaccines making them risky for patients with egg allergy; however, they contain only microscopic amounts of the protein and numerous studies have shown rate of adverse reactions to be no different in patients with versus without egg allergy in response to the inactivated influenza vaccine (11). Updated guidelines from the US Centers for Disease Control and Prevention and the American Academy of Pediatrics Committee on Infectious Diseases reflect this (12-14). Yellow fever virus vaccines are cultured in embryonated hen's eggs and are contraindicated for those with severe hen's egg allergy; however, if needed can be administered in graded fashion by an allergist $(15,16)$. Other culture media used besides hen's egg include horse serum, murine or simian cells, and canine kidney cells. Latex contamination can also be present from syringe plungers and vial stoppers. Vaccine stabilizers that can cause allergic reactions include gelatin, lactose, polysorbate $80 / 20$, and polygeline (12). Even the active vaccine agents themselves can very rarely cause allergic reactions (e.g., toxoids, attenuated pathogens) (7). Preservatives in various vaccines include thimerosal, formaldehyde, and 2-phenoxyethanol as a replacement for thimerosal. While a rare reaction, preservatives and other ingredients such as aluminum adjuvant can be triggers of T-cell-mediated contact dermatitis reactions that may be systemic after parenteral allergen administration (17-19). Typically, prior contact sensitization to these preservatives occurs through topical applications of products containing the ingredient.

Though rare, anaphylaxis is recognized as potentially occurring from a variety of vaccines and can result in death (20). Anaphylaxis is an acute systemic hypersensitivity reaction. Fatalities are typically from airway obstruction (bronchospasm, laryngeal edema) and/or cardiovascular collapse. Other symptoms may occur such as sneezing, rhinorrhea, vomiting, diarrhea, urticaria, itching, or sudden change in demeanor such as excessive irritability or clinging to caregiver in a non-verbal patient. Immediate treatment can quickly reverse these effects and prevent morbidity and mortality.

It is difficult to precisely identify the incidence of anaphylaxis after vaccination because it is so rare. Until recently, publications differed in their definitions of anaphylaxis but in 2006-2007 standard case definitions were established (21). A recent report estimates the rate of anaphylaxis to vaccines as 1.3 per 1,000,000 vaccine doses (22).

Anaphylaxis to the influenza, measles-mumps-rubella (MMR), varicella, or Japanese encephalitis vaccines are usually associated with gelatin sensitivity while anaphylactic reactions to hepatitis $B$ virus vaccine can be triggered by sensitivity to yeast (Saccharomyces cerevisiae) (12,23-25). Also rare but reported are anaphylaxis to meningococcal vaccines and the human papillomavirus vaccines (20). Anaphylaxis to the tetanus toxoid vaccine may be due to hypersensitivity to the tetanus toxoid antigens, aluminum phosphate, or thimerosal, if present (26). In cases of anaphylaxis suspected to be due to a vaccine, it is important to have close examination of the circumstances surrounding the reaction and possible evaluation by an allergist for further testing to determine specific ingredient allergy and further management. Additionally, anyone with reported allergies to a vaccine component should be evaluated by an allergist to determine if and how to give a vaccine containing said component to that individual. The person who develops a severe reaction for the first time to a vaccine is, of course, impossible to predict unless their history notes specific allergies (e.g., eggs, gelatin, yeast). However, in contrast to the many millions of lives saved by vaccination, there are a very few that are seriously injured by a vaccine because of severe immunological reaction $(13,27,28)$.

\section{Autoimmunity}

Another concern that has been raised is that various vaccines [i.e., MMR, DTaP, Haemophilus influenzae type B (HiB), and others] can lead to type 1 diabetes mellitus. Some speculate that molecular mimicry between viral antigens and self antigens is the mechanism, while others point to increased activation of macrophages $(29,30)$. However, larger, more rigorous studies have shown no credible evidence for such a link $(20,31)$. Postural orthostatic tachycardia syndrome (POTS) may be seen in unusual situations following the human papillomavirus vaccine and other vaccines $6-60$ or 
more days post-vaccination (32). Post-vaccination POTS may be an autoimmune condition due to the development of cross-reacting autoantibodies and can be managed with a variety of measures including reducing hypovolemia and increasing blood pressure by increasing salt as well as oral fluid intake and selective pharmacotherapy (i.e., desmopressin).

Transient arthralgia has been identified in females receiving the MMR vaccine and is linked to the rubella constituent (20). Controversy has existed regarding arthritis and the rubella vaccine since its first development in the 1960s and the addition into the combined MMR vaccine in 1971. Reports emerged noting some females develop acute and chronic arthritis after receiving a rubella vaccine. Some of this concern arose from reviewing information from the US Vaccine Adverse Event Reporting System (VAERS) and in 1991 the US Institute of Medicine (IOM) noted that up to $15 \%$ of adult females who received this vaccine developed acute arthritis (33). The US Court of Federal Claims has accepted some claims from patients of a link between the rubella vaccine and chronic arthropathy based on cases submitted to the US National Vaccine Injury Compensation Program (VICP) (34).

More recent evaluation of rubella vaccine effects does not suggest a link with acute or chronic arthritis but confirms a link with transient arthralgia. A recent review of VAERS data from 2003 to 2013 noted rash in $17 \%$, pain in $13 \%$, and arthralgia in $13 \%$ of those receiving the MMR vaccine (35). A 2011 IOM report concluded that there was evidence for the development of transient arthralgia in women and children but there was not enough sound scientific evidence to accept or reject a causal link between the rubella vaccine and chronic arthritis in women and children (20).

The fact that natural rubella virus infection can cause musculoskeletal symptoms including arthritis only adds to the cloudiness of the picture. This example also illustrates some of the dilemmas that emerge from conclusions based on the VAERS and VICP reports. Research on children with juvenile idiopathic arthritis who had undergone primary immunization showed that MMR booster vaccination was immunogenic and did not lead to any deterioration of arthritis symptoms (36). Also, it is clear that proper application of rubella vaccination prevents the congenital rubella syndrome and protects children from significant morbidity and mortality (37).

Other vaccines (i.e., hepatitis B vaccine) have been anecdotally linked with rare cases of arthritis but careful evaluation reveals no actual etiological link (38). The possibility cannot be excluded that transient immune complexes could form in response to hepatitis B antigens in the vaccine, leading to inflammatory reaction in tissues (including joints), similar to a serum sickness-like condition in patients with acute hepatitis B infection. Infection can induce a variety of autoimmune phenomena and controversy continues regarding the role that immunization may have due to such potential issues as polyclonal activation (adjuvant reaction) or molecular mimicry (29).

\section{Guillian-Barré syndrome}

The previous consideration of Guillian-Barré syndrome (GBS) and influenza vaccination is an example of this potential phenomenon. GBS is an acute polyradiculopathy usually described as an autoimmune disorder following an upper respiratory or gastrointestinal infection in a susceptible person. This peripheral nerve disorder was found to have increased incidence in those vaccinated with the 1976-1977 swineinfluenza vaccine (A/New Jersey) and resulted in the temporary suspension of the US National Influenza Immunization Program on December 16, 1976 (39). A careful analysis of the situation revealed GBS in approximately 1 in 100,000 individuals vaccinated with this particular vaccine, and the increased risk for GBS was mainly within 5 weeks of vaccination, though up to 9-10 weeks in some (39). During the 1976-1977 National Influenza Program 58 people died from GBS and 32 of them $(58 \%)$ had been given the $\mathrm{A} / \mathrm{New}$ Jersey influenza vaccine; most of these deaths were from respiratory paralysis.

An intensive surveillance program was developed to study this potential link and many subsequent reports of vaccination with other strains (for example, the 19781979 and the 2009-2010 influenza vaccines, including monovalent and trivalent) did not reveal a statistically significant increase in risk for this syndrome (39-43). In one database of 3 million people studied between 1995 and 2006 there were 415 cases of GBS, but no cases of recurrent GBS after influenza vaccination and no cases of GBS within 6 weeks of receiving any vaccine $(40,41)$.

This potential link remains under passive as well as active surveillance and some studies, such as those of the influenza A (H1N1) 2009 monovalent inactivated vaccine, suggest a low risk of GBS with influenza vaccinationestimated at 1.6 excess cases per million vaccinations (42). The shadow of the 1976-1977 National Influenza Program 
remains and the US Advisory Committee on Immunization Practices recommends not giving an influenza vaccine to an individual with a history of GBS within 6 weeks of a prior influenza vaccination unless they are at high risk for severe complications from an influenza illness (41). However, the risk of GBS related to influenza vaccination is thought to be lower than that of GBS related to wild type influenza strain infection (43). Again, it is difficult to be precise in this situation since, though GBS is the most common cause of acute flaccid paralysis, it is a rare disorder and even rarer after influenza vaccination.

\section{Neurologic adverse effects}

Severe neurological disease is a rare complication of vaccine administration and typically involves a patient with immunocompromised status and/or underlying neurological disease $(44,45)$. Varicella vaccine can, in rare cases, lead to disseminated varicella infection in immunocompromised persons with eventual meningitis, pneumonia, or hepatitis, and vaccine strain viral reactivation leading to meningitis or encephalitis $(20,46)$. Rare cases are reported of MMRinduced measles inclusion body encephalitis in persons with immunocompromised status from the measles component of the vaccine $(20,47)$.

Some vaccines have been associated with fever and resultant febrile seizures, including MMR, DTaPinactivated poliovirus-HiB combination vaccine (DTaPIPV-HiB), and combination MMR with varicella $(\mathrm{MMR}+\mathrm{V})$ (48-50). Febrile seizures are typically not a severe condition and do not increase risk of epilepsy (48). Of note, the increased risk of febrile seizures with $M M R+V$ is not seen with MMR and varicella vaccines given separately during the same visit, or in children age 4 years or older (50).

In unusual circumstances epilepsy may occur around the time of vaccination; however, genetic or structural defects are the cause in most of these situations. In a study of 165 claims of vaccine injury from seizure disorder and/ or encephalopathy reported to the VICP between 1995 and 2005, a significant number of patients had pre-existing neurologic or neurodevelopmental abnormalities; many of those with chronic epilepsy had histories consistent with genetically-caused epilepsy such as severe myoclonic epilepsy of infancy (SCN1A-related Dravet syndrome), tuberous sclerosis, or cerebral dysgenesis (45). Other underlying issues that may cause seizures around the time of vaccination include epileptic encephalopathy and benign epilepsy (51). Similarly, there is a commonly-held belief that the whole-cell pertussis vaccine of the $20^{\text {th }}$ century caused infantile encephalopathy (3). Despite refutation of such a link between this vaccine and severe neurological disease by expert researchers of infectious diseases (52), acellular pertussis vaccines were developed and first licensed in the United States in 1991. Today some researchers call these fears "only partially justified" (53) and others have identified an inherited defect of the $S C N L A$ gene as a major cause of the rare infantile encephalopathy in infants receiving the whole-cell pertussis vaccine (54).

Further genetic research may be able to improve predictions of which few patients will have neurological sequelae from vaccinations (45). The risk of vaccine-induced epilepsy or encephalopathy in children with epilepsy is very low and severe long-term sequelae are low in occurrence. Recent data has linked increased risk of narcolepsy with H1N1 vaccination, though it is unclear if this is a true causal link or a hastening of disease in susceptible persons (55). Concerns that the inactivated influenza vaccine induces Bell's palsy were not proven when carefully examined (20). These examples highlight the need for ongoing research and critical evaluation of the available data rather than anecdotal reports. Anecdotal reports of neurological complications from vaccines typically are reporting a temporal and not causal link; one should be cautious in stating an etiologic link unless proven by appropriate, confirmed scientific research (56).

\section{Autism}

One of the greatest myths against vaccine science of the $20^{\text {th }}$ century that still lingers in the $21^{\text {st }}$ century has been the persistent assertion that the MMR vaccine, typically the measles component, causes autism in young children. There are many factors at work in this claim based on historical underpinnings of two centuries of anti-vaccine views, parental anger at false claims by health care professionals (HCPs) for decades (1940s to 1980s plus) blaming autism on "cold" mothers, and confusion of parents and HCPs between temporal versus etiologic associations $(2,3)$. Parents would see their young children receive the MMR vaccine and be diagnosed within 1-2 years with autism $(2,3)$.

A now-retracted paper was published in Lancet in 1998 linking autism to the MMR vaccine by claiming it causes "autistic enterocolitis". That work fueled the hysteria against vaccines, mercury, and vaccine components $(2,3)$. The author's status as a registered physician in the UK was revoked. Additionally, since that time a number of studies 
have carefully looked at the potential link between the MMR vaccine, other vaccines, mercury or other vaccine adjuvants, and autism and found no plausible scientific evidence to support this myth $(2,20,57)$. Despite all this, people continue to cite such fears as reasons they avoid vaccinating their children.

\section{Other adverse effects}

\section{Rashes/non-hypersensitivity skin reactions}

Severe skin reactions have been rarely noted in vaccine recipients. Severe dermatological inflammation may result if some vaccines are administered subcutaneously or intradermally instead of receiving a deep intramuscular injection. For example, a 4-month-old baby developed dermal necrotizing granulomatous giant cell reaction at the site of a presumed improper injection of 13-valent pneumococcal conjugate vaccine; this severe inflammatory reaction was only present at the second dose and not the first or third dose where proper injection was presumably accomplished (58).

Varicella vaccination can result in rash at the site of vaccination, and rarely, the vaccine strain virus can be reactivated months or years after vaccination and cause rash as well (20).

\section{Bursitis}

There are a number of reports of deltoid bursitis postvaccine injection into the deltoid muscle, causing temporary shoulder pain and loss of shoulder movement (17). Publications in this regard suggest that vaccine injection into the sub-deltoid bursa or sub-acromial bursa can induce this condition and can be prevented by avoiding the upper third of the deltoid muscle for vaccine injection (59). Corticosteroid injections into the inflamed bursa(e) will resolve this preventable situation. Also rarely reported is limb swelling, pain, and erythema that begins within a day of a vaccine injection; it can be seen at all ages with various vaccines (60). This swelling can involve the proximal and distal parts of the extremity and is managed with analgesics; spontaneous resolution is typically seen within 24 hours (60).

\section{Syncope}

It is well-known that injection of various substances, including vaccines, can lead to vasovagal syncope, particularly in adolescent females $(20,61)$. It is possible for any post-syncopal fall to lead to an injury of varying severity. Clinicians should be aware of this and observing the person post-vaccination for a period of time (i.e., 15 minutes) is suggested by some authorities-though the syncope can occur well after this 15 -minute timeframe.

\section{Intussusception}

Use of rotavirus vaccine was temporarily suspended in the US in 1999 because of unacceptably high rates of intussusception found in infants administered this vaccine. Anti-vaccine forces seized on this suspension noting it supports their claims that vaccines are harmful. However, intense research on this link occurred and rotavirus administration was restarted in 2006 with the two currently available vaccines. Though some studies note no increased risk of intussusception in infants receiving a rotavirus vaccine, others have found an excess intussusception case load of 1.5 per 100,000 infants given the first dose of the pentavalent vaccine (62). A history of intussusception and a history of severe combined immunodeficiency are currently contraindications to administration of rotavirus vaccine to an infant (63).

\section{Proposed effects on social mores}

Confusion and concern over the human papillomavirus (HPV) vaccine has been present since the introduction of HPV vaccination in 2006. This vaccine has been plagued with false accusations of being ineffective, dangerous, or conducive to teenage promiscuity. Studies have shown these statements to be false and the vaccine has proven effective despite parental resistance to its utilization (64). Though it promises to prevent the majority of the 530,000 cervical cancers worldwide that represent the second most common cause of cancer deaths in women, and prevents oropharyngeal, penile, and anal cancers, attempts to legally mandate this vaccine caused considerable uproar perhaps similar to that noted with attempts to mandate smallpox vaccine in England with the 1853 Vaccination Act $(2,3)$. It is problematic that a vaccine that can prevent sexually transmitted disease is not accepted by a society that does not provide comprehensive sex education to its children (65).

\section{Death from vaccines}

Vaccine-induced death is a very rare event in the $21^{\text {st }}$ century 
if recommendations for vaccine administration are carefully followed. Vaccine-induced death has mainly occurred with the smallpox vaccine and is historically noted with other vaccines (27). It is difficult to link death of a person with receiving a specific vaccine due to the inherent principle that correlation does not necessarily indicate causation-death may have been from unrelated or unknown causes. Out of hundreds of millions of vaccinations given in the United States from 1997-2013, there were 2,149 deaths reported to VAERS that included 1,244 child reports of death and 526 adult reports (28). Extrapolating the exact cause of death from VAERS is problematic and non-scientific. However, when a person dies shortly after receiving a vaccine there is concern by the family, fueled by common vaccine safety myths, that the vaccine was directly responsible. The causes of these deaths reported were consistent with typical causes of death in the general population and there were rare reports of death directly due to a specific vaccine.

Given that typical recommended immunization schedules start under the age of 6 months, and that is also an age of high sudden infant death syndrome (SIDS), some people believe that vaccines are tied to increased risk of SIDS or are directly causative. However, multiple studies and metaanalyses have shown this not to be the case, and in fact some studies have shown vaccination to be associated with a lower risk of SIDS (66).

In the rare cases of direct link between vaccine and patient death, cause of death can include anaphylaxis, generalized infection with vaccine-strain in a severely immune-compromised individual receiving a live viral vaccine, respiratory failure due to paralytic poliomyelitis (from vaccine-strain infection from oral poliovirus vaccine) or GBS, post-vaccine syncope leading to a fall with injury, post-rotavirus vaccine intussusception, complications of yellow fever vaccine (e.g., viscerotropic disease or neurologic disease), and complications of the smallpox vaccine (i.e., progressive vaccinia, eczema vaccinatum, post-vaccinal encephalitis, myocarditis, or dilated cardiomyopathy) (67).

\section{Conclusions}

Severe adverse reactions to vaccines may occur but are fortunately rare and do not remove the conclusion that immunizations remain one of the paramount medical advances of human history $(1-3,57,68)$. Contraindications and precautions for all and specific vaccines must be understood and carefully followed by clinicians. Temporary contraindications to vaccines include moderate to severe illness (as determined by the clinician) and also, in the case of live attenuated vaccines, pregnancy and immunosuppression.

Unusual but severe reactions to vaccines are considered in this discussion. Known injection-related phenomena include syncope and deltoid bursitis. There is no link between vaccines and autism, diabetes mellitus, or promiscuous behavior. Consultation with experts in allergy and immunology is beneficial in application of live attenuated vaccines to those with immunocompromised status or in cases of unclear reaction to a vaccine.

\section{Acknowledgments}

The authors wish to acknowledge our department chair, Dr. Dilip Patel, for his support of all our scholarly works, including this article.

\section{Footnote}

Conflicts of Interest: The authors have no conflicts of interest to declare.

Ethical Statement: The authors are accountable for all aspects of the work in ensuring that questions related to the accuracy or integrity of any part of the work are appropriately investigated and resolved.

\section{References}

1. Whitney CG, Zhou F, Singleton J, et al; Centers for Disease Control and Prevention (CDC). Benefits from immunizations during the vaccines for children era-United States, 1994-2013. MMWR Morb Mortal Wkly Rep 2014;63:352-5.

2. Greydanus DE, Toledo-Pereyra LH. Historical perspectives on autism: its past record of discovery and its present state of solipsism, skepticism, and sorrowful suspicion. Pediatr Clin North Am 2012;59:1-11.

3. Greydanus DE. Historical perspectives on the antaean animadversion of vaccine science in the 21 st century. J Clin Trials 2013;3:140.

4. Ten threats to global health in 2019. Geneva, Switz: World Health Organization; 2019. Available online: www.who. int/emergencies/ten-threats-to-global-health-in-2019. Accessed 2019 Mar 25.

5. Bankamp B, Hickman C, Icenogle JP, Rota PA. Successes and challenges for preventing measles, mumps and rubella 
by vaccination. Curr Opin Virol 2019;34:110-6.

6. Measles Cases and Outbreaks. Centers for Disease Control and Prevention; 2019. Available online: https://www.cdc. gov/measles/cases-outbreaks.html. Accessed 2019 Mar 25.

7. Vanlander A, Hoppenbrouwers K. Anaphylaxis after vaccination of children: review of literature and recommendations for vaccination in child and school health services in Belgium. Vaccine 2014;32:3147-54.

8. Rajan TV. The Gell-Coombs classification of hypersensitivity reactions: a re-interpretation. Trends Immunol 2003;24:376-9.

9. Pichichero ME, Casey JR. Acellular pertussis vaccines for adolescents. Pediatr Infect Dis J 2005;24:S117-26.

10. Siegrist CA. Mechanisms underlying adverse reactions to vaccines. J Comp Pathol 2007;137 Suppl 1:S46-50.

11. Greenhawt M, Turner PJ, Kelso JM. Administration of influenza vaccines to egg allergic recipients: A practice parameter update 2017. Ann Allergy Asthma Immunol 2018;120:49-52.

12. Fritsche PJ, Helbling A, Ballmer-Weber BK. Vaccine hypersensitivity-update and overview. Swiss Med Wkly 2010;140:238-46.

13. Grohskopf LA, Sokolow LZ, Broder KR, et al. Prevention and control of seasonal influenza with vaccines: recommendations of the advisory committee on immunization practices-United States, 2017-2018 influenza season. MMWR Recomm Rep 2017;66:1-20.

14. Committee on Infectious Diseases. Recommendations for prevention and control of influenza in children, 20172018. Pediatrics 2017;140:e20172550.

15. Muñoz-Cano R, Sanchez-Lopez J, Bartra J, et al. Yellow fever vaccine and egg allergy: really a problem? Allergy 2010;65:533-4.

16. Rutkowski K, Ewan PW, Nasser SM. Administration of yellow fever vaccine in patients with egg allergy. Int Arch Allergy Immunol 2013;161:274-8.

17. Kuritzky LA, Pratt M. Systemic Allergic Contact Dermatitis After Formaldehyde-Containing Influenza Vaccination. J Cutan Med Surg 2015;19:504-6.

18. Rosenblatt AE, Stein SL. Cutaneous reactions to vaccinations. Clin Dermatol 2015;33:327-32.

19. Descatha A. Unusual delayed reaction after $H 1 N 1$ vaccine. Asian Pac J Allergy Immunol 2010;28:302-3.

20. Adverse effects of vaccines: evidence and causality. Institute of Medicine of the National Academies. August, 2011. Available online: http://www.vaccinesafety.edu/IOMReports.htm. Accessed 5/15/2019.

21. McNeil MM, DeStefano F. Vaccine-associated hypersensitivity. J Allergy Clin Immunol 2018;141:463-72.

22. McNeil MM, Weintraub ES, Duffy J, et al. Risk of anaphylaxis after vaccination in children and adults. J Allergy Clin Immunol 2016;137:868-78.

23. Coop CA, Balanon SK, White KM, et al. Anaphylaxis from the influenza virus vaccine. Int Arch Allergy Immunol 2008;146:85-8.

24. DiMiceli L, Pool V, Kelso JM, et al. Vaccination of yeast sensitive individuals: review of safety data in the US vaccine adverse event reporting system (VAERS). Vaccine 2006;24:703-7.

25. Pool V, Braun MM, Kelso JM, et al. Prevalence of antigelatin IgE antibodies in people with anaphylaxis after measles-mumps-rubella vaccine in the United States. Pediatrics 2002;110:e71.

26. Das S, Mondal S. Tetanus toxoid induced anaphylaxis. J Vaccines Vaccin 2012;3:126.

27. Belongia EA, Naleway AL. Smallpox vaccine: the good, the bad, and the ugly. Clin Med Res 2003;1:87-92.

28. Moro PL, Arana J, Cano M, et al. Deaths reported to the Vaccine Adverse Event Reporting System, United States, 1997-2013. Clin Infect Dis 2015;61:980-7.

29. Shoenfeld Y, Aron-Maor A. Vaccination and autoimmunity-'vaccinosis': a dangerous liaison? J Autoimmun 2000;14:1-10.

30. Classen JB, Classen DC. Clustering cases of insulin dependent diabetes (IDDM) occurring three years after Hemophilus influenza B (HiB) immunization support causal relationship between immunization and IDDM. Autoimmunity 2002;35:247-53.

31. Demicheli V, Rivetti A, Debalini MG, et al. Vaccines for measles, mumps and rubella in children. Cochrane Database Syst Rev 2012;2:CD004407.

32. Blitshteyn S. Postural tachycardia syndrome following human papillomavirus vaccination. Eur J Neurol 2014;21:135-9.

33. Geier DA, Geier MR. Rubella vaccine and arthritis adverse reactions: an analysis of the Vaccine Adverse Events Reporting System (VAERS) database from 1991 through 1998. Clin Exp Rheumatol 2001;19:724-6.

34. Weibel RE, Benor DE. Chronic arthropathy and musculoskeletal symptoms associated with rubella vaccines. A review of 124 claims submitted to the National Vaccine Injury Compensation Program. Arthritis Rheum 1996;39:1529-34.

35. Sukumaran L, McNeil MM, Moro PL, et al. Adverse events following measles, mumps, and rubella vaccine in adults reported to the Vaccine Adverse Event 
Reporting System (VAERS), 2003-2013. Clin Infect Dis 2015;60:e58-65.

36. Heijstek MW, Kamphuis S, Ambrust W, et al. Effects of the live attenuated measles-mumps-rubella booster vaccination on disease activity in patients with juvenile idiopathic arthritis: a randomized trial. JAMA 2013;309:2449-56.

37. Martínez-Quintana E, Castillo-Solórzano C, Tomer N, et al. Congenital rubella syndrome: a matter of concern. Rev Panam Salud Publica 2015;37:179-86.

38. Systemic adverse effects of hepatitis $B$ vaccines are rare. Prescrire Int 2004;13:218-23.

39. Hurwitz ES, Schonberger LB, Nelson DB, et al. GuillainBarré syndrome and the 1978-1979 influenza vaccine. N Engl J Med 1981;304:1557-61.

40. Baxter R, Bakshi N, Fireman B, et al. Lack of association of Guillain-Barré syndrome with vaccinations. Clin Infect Dis 2013;57:197-204.

41. Baxter R, Lewis N, Bakshi N, et al.; CISA Network. Recurrent Guillain-Barre syndrome following vaccination. Clin Infect Dis 2012;54:800-4.

42. Salmon DA, Proschan M, Forshee R, et al. Association between Guillain-Barré syndrome and influenza A (H1N1) 2009 monovalent inactivated vaccines in the USA: a metaanalysis. Lancet 2013;381:1461-8.

43. Vellozzi C, Igbal S, Broder K. Guillain-Barre syndrome, influenza, and influenza vaccination: the epidemiologic evidence. Clin Infect Dis 2014;58:1149-55.

44. Sejvar JJ. Vaccines and neurologic disease. Semin Neurol 2011;31:338-55.

45. Lateef TM, Johann-Liang R, Kaulas H, et al. Seizures, encephalopathy, and vaccines: experience in the National Vaccine Injury Compensation Program. J Pediatr 2015;166:576-81.

46. Galea SA, Sweet A, Beninger P, et al. The safety profile of varicella vaccine: a 10 -year review. J Infect Dis 2008;197:S165-9.

47. Bitnun A, Shannon P, Durward A, et al. Measles inclusionbody encephalitis caused by the vaccine strain of measles virus. Clin Infect Dis 1999;29:855-61.

48. Vestergaard M, Hviid A, Madsen KM, et al. MMR vaccination and febrile seizures: evaluation of susceptible subgroups and long-term prognosis. JAMA 2004;292:351-7.

49. Sun Y, Christensen J, Hviid A, et al. Risk of febrile seizures and epilepsy after vaccination with diphtheria, tetanus, acellular pertussis, inactivated poliovirus, and Haemophilus influenzae type B. JAMA 2012;307:823-31.
50. Klein NP, Lewis E, Baxter R, et al. Measles-containing vaccines and febrile seizures in children age 4 to 6 years. Pediatrics 2012;129:809-14.

51. Verbeek NE, Jansen FE, Vermeer-de Bondt PE, et al. Etiologies for seizures around the time of vaccination. Pediatrics 2014;134:658-66.

52. Cherry JD. 'Pertussis vaccine encephalopathy': It is time to recognize it as the myth that it is. JAMA 1990;263:1679-80.

53. Greco D, Esposito S, Tozzi A, et al. Whooping cough, twenty years from acellular vaccines introduction. Ann Ig 2015;27:415-31.

54. Shorvon S, Berg A. Pertussis vaccination and epilepsyan erratic history, new research and the mismatch between science and social policy. Epilepsia 2008;49:219-25.

55. Thebault S, Vincent A, Gringras P. Narcolepsy and H1N1 vaccination: a link? Curr Opin Pulm Med 2013;19:587-93.

56. Miravalle AA, Schreiner T. Neurologic complications of vaccinations. Handb Clin Neurol 2014;121:1549-57.

57. Spencer JP, Trondsen Pawlowski RH, Thomas S. Vaccine adverse events: separating myth from reality. Am Fam Physician 2017;95:786-94.

58. Alsuwaidi AR, Albawardi A, Khan NH, et al. A 4-month-old baby presenting with dermal necrotizing granulomatous giant cell reaction at the injection of 13 valent pneumococcal conjugate vaccine: a case report. J Med Case Rep 2014;8:285.

59. Cook IF. Subdeltoid/subacromial bursitis association with influenza vaccination. Hum Vaccin Immunother 2014;10:605-6.

60. van Balveren-Slingerland L, Rümke HC, Kant AC. Reported adverse events following influenza vaccination. Ned Tijdschr Geneeskd 2014;158:A6841. Dutch.

61. Centers for Disease Control and Prevention (CDC). Syncope after vaccination-United States, January 2005July 2007. MMWR Morb Mortal Wkly Rep 2008;57:45760.

62. Shui IM, Baggs J, Patel M, et al. Risk of intussusception following a pentavalent rotavirus vaccine in US infants. JAMA 2012;307:598-604.

63. Centers for Disease Control and Prevention (CDC). Addition of history of intussusception as a contraindication for rotavirus vaccination. MMWR Morb Mortal Wkly Rep 2011;60:1427.

64. Kash N, Lee MA, Kollipara R, et al. Safety and efficacy data on vaccines and immunization to human papillomavirus. J Clin Med 2015;4:614-33.

65. Lindau ST, Tetteh AS, Kasza K, et al. What schools teach 
our patients about sex: content, quality, and influences on sex education. Obstet Gynecol 2008;111:256-66.

66. Vennemann MM, Höffgen M, Bajanowski T, et al. Do immunisations reduce the risk for SIDS? A meta-analysis. Vaccine 2007;25:4875-9.

67. Miller ER, Moro PL, Cano M, et al. Deaths following

Cite this article as: Greydanus DE, Leonov A, Elisa A, Azmeh R. Should rare immunologic, neurologic, and other adverse events be indications to withhold vaccination? Transl Pediatr 2019;8(5):419-427. doi: 10.21037/tp.2019.06.01 vaccination: what does the evidence show? Vaccine 2015;33:3288-92.

68. Vetter V, Denizer G, Friedland LR, et al. Understanding modern-day vaccines: what you need to know. Ann Med 2018;50:110-20. 